\title{
Exotics in leptonic machines
}

\author{
Zhiqing LIU*广 \\ Johannes Gutenberg University Mainz \\ E-mail: पiuzduni-mainz.de
}

Exotic states with quark content different from conventional baryons and mesons are of high interest in the hadron spectroscopy field. Over the last decade, more than a dozen of $X Y Z$ particles - good candidates for exotic states - were discovered experimentally, of which leptonic machines play an extremely important role. The $X(3872)$ is the first exotic state which was discovered by Belle in 2003, and by now still remains quite puzzling for its nature. Following the $X(3872)$, there are also quite mysterious $1^{--} Y$-family observed by $B A B A R$ and Belle. Recently, the BESIII experiment takes more than $5 \mathrm{fb}^{-1}$ data above $4 \mathrm{GeV}$, which also enable us to study the $X Y Z$ particles efficiently. In 2013, BESIII Collaboration discovered the charged charmoniumlike states $Z_{c}(3900)$, which is a convincing exotic state with at least four quarks inside. In addition, BESIII also has studied $X(3823), X(3872)$, and $Y(4260)$ in an unique way. In this talk, I'll review the recent progress on exotic states with leptonic machines.

Flavor Physics \& CP Violation 2015

May 25-29, 2015

Nagoya, Japan

\footnotetext{
* Speaker.

${ }^{\dagger}$ I would like to thank the Marie Curie International Incoming Fellowship within Seventh Framework Programme of the European Union under Grant Agreement No. 627240 for the support of my work and travel.
} 


\section{Introduction}

By decades, people know from the quark model [W] that hadron matter existing in our universe is composed of 3 quarks (baryon) or quark anti-quark pairs (meson). However, QCD (the theory to describe strong force, which bind quarks together), allows new forms of hadron matter in our universe, such as multi-quark states, hybrid states, glue balls and so on. Such kind of new hadrons are referred as exotic states. Although exotic states are expected from the theoretical point of view, we are still lack of convincing evidence for them experimentally. Only until the last ten years, lots of new particles in the charmonium (bound state of charm quark and anti-charm quark) mass region were observed. These new particles show different features with normal charmonium states, and are good candidates for exotic states (they are called charmoniumlike states or $X Y Z$ particles).

In 2003, the Belle experiment observed a new charmoniumlike state $X(3872)$ [వ], which is the first candidate for a four quark state. Later, the BABAR experiment observed another new vector

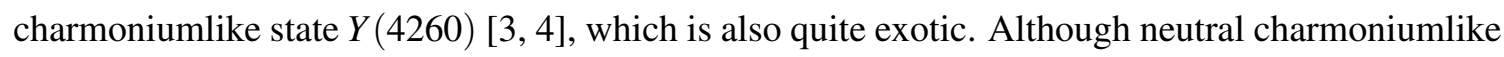
states are promising candidates for exotic hadrons, there is still big challenge in how to distinguish them from conventional charmonium states. However, this ambiguous situation has been changed since 2013, when a new charged charmoniumlike state $Z_{c}(3900)$ discovered by the BESIII Collaboration [罒], and immediately confirmed by Belle Collaboration [司] and CLEO-c's data [ $\square$ ]. $Z_{c}(3900)$ carries electric charged, which obvious can not be normal charmonium. The minimal quark content of $Z_{c}(3900)$ should be a four quarks combination [ 8$]$.

The exciting discoveries of $X Y Z$ particles have stimulated worldwide efforts in studying them, among which, the leptonic machines play a significant role.

\section{The vector $Y$-family}

The leading-order hadron production in a leptonic machine is $e^{+} e^{-} \rightarrow \gamma^{*} \rightarrow q \bar{q}$, i.e. through single photon annihilation process. Thus, the direct produced hadron state via $e^{+} e^{-}$annihilation shares the same quantum number with photon, i.e. $J^{P C}=1^{--}$. Exotic state which was produced in such kind of way was usually called $Y$-state. There are two typical approaches to study $Y$-state in a $e^{+} e^{-}$machine, via direct production (scanning experiment such as BESIII, CLEO-c) and initialstate-radiation (B-factories such as $B A B A R$ and Belle). Figure $\square$ shows the luminosity collected by BESIII and the equivalent initial-state-radiation (ISR) luminosity in the charmonium energy region of Belle by taking $967 \mathrm{fb}^{-1}$ data at (or near) $\sqrt{s}=10.58 \mathrm{GeV}$.

Using the ISR technique, the BABAR experiment studied the $e^{+} e^{-} \rightarrow \gamma_{I S R} \pi^{+} \pi^{-} J / \psi$ process, and observed a new resonance $Y(4260)$ [ [B]]. The same process has been studied by Belle, instead of one resonance, Belle observed two resonances $Y(4008)$ and $Y(4260)$ [䧃]. With more statistics, both $B A B A R$ and Belle updated their measurements. Figure $\square$ shows the recent measurements from BABAR and Belle. Both experiments confirmed the $Y(4260)$ resonance (BABAR reported mass $4245 \pm 5$ (stat) \pm 4 (sys) $\mathrm{MeV} / c^{2}$ and width $114_{-15}^{+16}$ (stat) \pm 7 (sys.) $\mathrm{MeV}$ for $Y(4260$ ), while Belle reported mass $4258.6 \pm 8.3$ (stat) \pm 12.1 (sys.) $\mathrm{MeV} / c^{2}$ and width $134.1 \pm 16.4$ (stat) \pm 5.5 (sys.) MeV), but show a discrepancy for the $Y(4008)$ state. The Belle experiment still shows an enhancement near $4 \mathrm{GeV}$, which is consistent with $Y(4008)$ [ [6]. Whereas, the BABAR experiment observed an increasing exponential shape near $4 \mathrm{GeV}[\mathrm{G}]$. The good news is that the BESIII ex- 

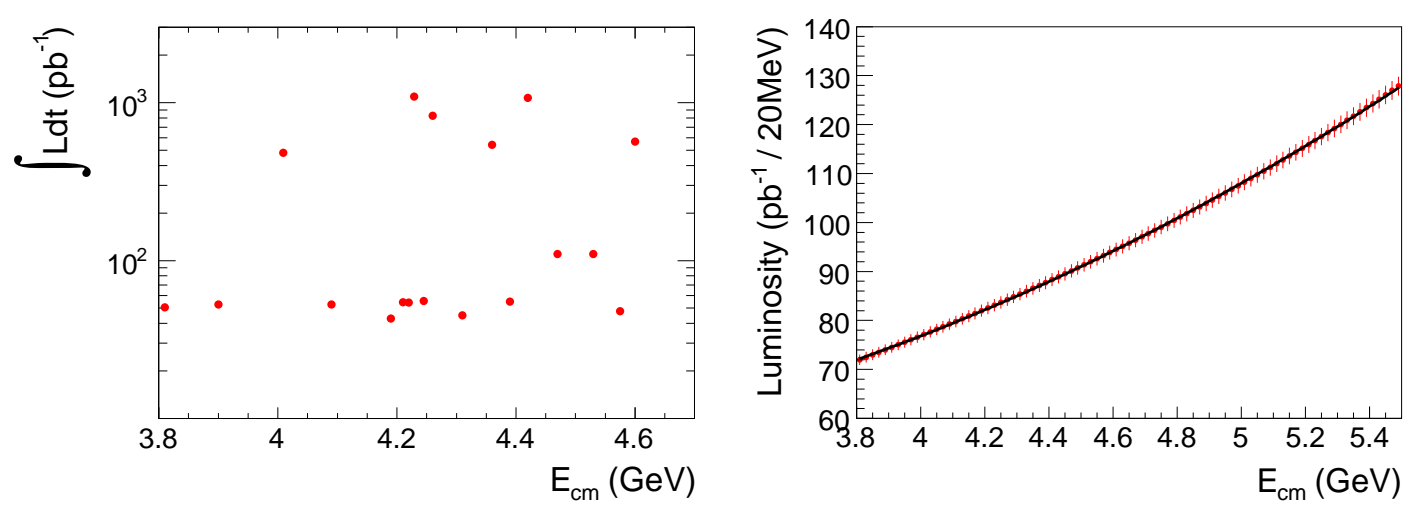

Figure 1: The integrated luminosity collected by BESIII (left) and equivalent ISR luminosity by Belle with $967 \mathrm{fb}^{-1}$ data at (or near) $\sqrt{s}=10.58 \mathrm{GeV}$ (right).

periment has already accumulated lots of scanning data sets in this energy region (c.f. Fig. 田), and might be able to settle down the controversy in the near future. With the similar method, the Belle experiment has studied $e^{+} e^{-} \rightarrow \gamma_{I S R} \pi^{+} \pi^{-} \psi(2 S)$ process. Two vector resonances $Y$ (4360) (mass $4361 \pm 9 \pm 9 \mathrm{MeV} / c^{2}$ and width $74 \pm 15 \pm 10 \mathrm{MeV}$ ) and $Y(4660)$ (mass $4664 \pm 11 \pm 5 \mathrm{MeV} / c^{2}$ and width $48 \pm 15 \pm 3 \mathrm{MeV}$ ) have been observed [ए]]. BABAR also updated the same process with its full luminosity, and the results agree with Belle's observation [Ш]], as seen in Fig. ㅁ.
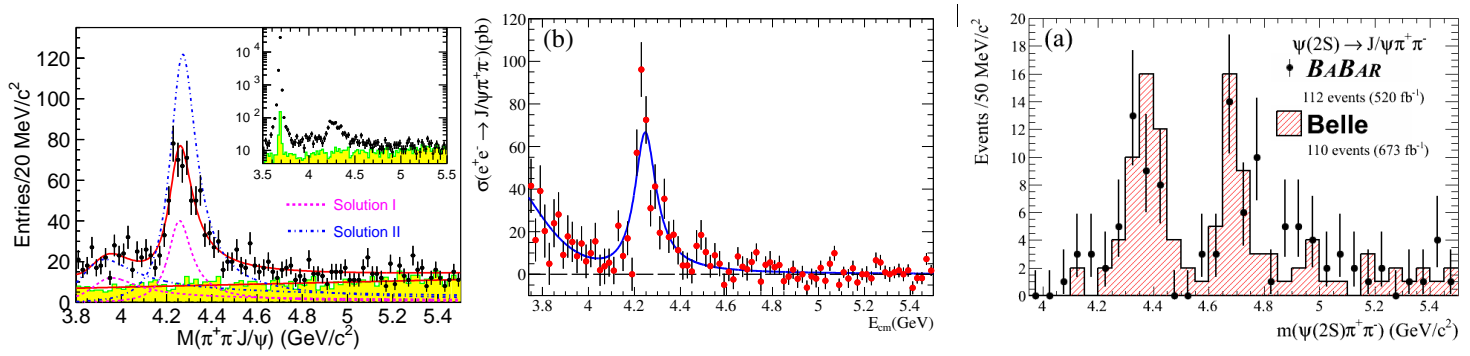

Figure 2: The $e^{+} e^{-} \rightarrow \pi^{+} \pi^{-} J / \psi$ mass distribution by Belle (left) and cross section by BABAR (middle); and $e^{+} e^{-} \rightarrow \pi^{+} \pi^{-} \psi(2 S)$ mass distribution by Belle and BABAR (right) with ISR method.

With the scanning data sets taken between 3.8 and $4.6 \mathrm{GeV}$, the BESIII experiment was able to study vector $Y$-state in direct $e^{+} e^{-}$annihilation. Using about $2.7 \mathrm{fb}^{-1}$ data taken between 4.21 and $4.42 \mathrm{GeV}$, the BESIII experiment has studied the $e^{+} e^{-} \rightarrow \omega \chi_{c 0}$ process. Significant $\omega \chi_{c 0}$ events has been observed at 4.23 and $4.26 \mathrm{GeV}$, with measured Born production cross section $(55.4 \pm 6.0 \pm$ $5.9) \mathrm{pb}$ and $(23.7 \pm 5.3 \pm 3.5) \mathrm{pb}$, respectively [12]. By studying the energy dependent production cross section, BESIII finds it is inconsistent with the line shape of the $Y(4260)$ resonance, which hints for new resonant structure. Assuming the $\omega \chi_{c 0}$ events come from a single resonance, BESIII extract the mass and width of the resonance to be $4230 \pm 8 \pm 6 \mathrm{MeV} / c^{2}$ and $38 \pm 12 \pm 2 \mathrm{MeV}$, and the statistical significance more than $9 \sigma$. Figure 3 shows the energy dependent cross section line shape of $e^{+} e^{-} \rightarrow \omega \chi_{c 0}$, and the corresponding fit assuming it's from Breit-Wigner resonant line shape. In addition to $\omega \chi_{c 0}$, both Belle and BESIII have investigated other exclusive channels.

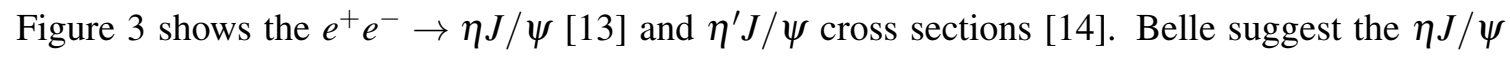
process comes from $\psi(4040)$ and $\psi(4160)$ decay. BESIII's cross section is consistent with Belle 
within errors, but shows possible enhancement near $4.2 \mathrm{GeV}$, which might be similar with the $\omega \chi_{c 0}$ case. For $\eta^{\prime} J / \psi$, the current precision from BESIII is low and it seems featureless. More data is needed to investigate whether there is possible structure existing.
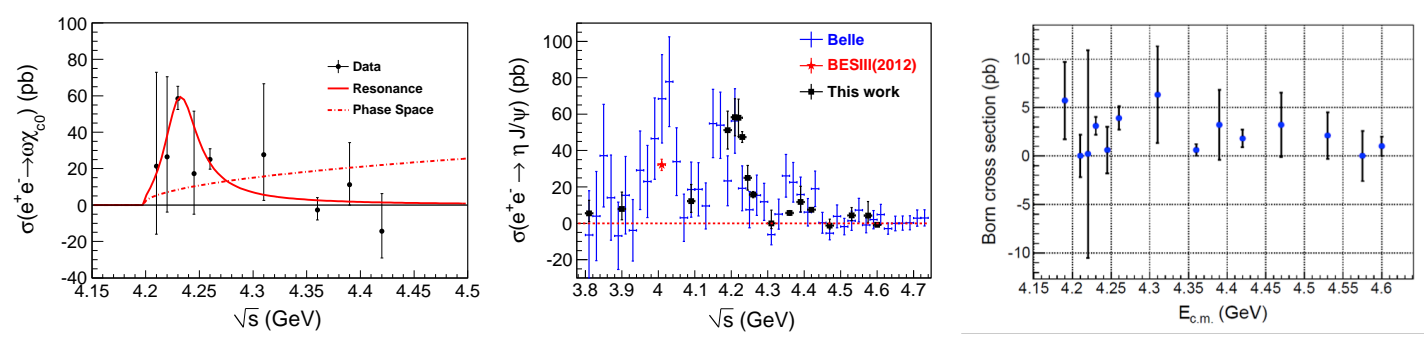

Figure 3: The measured energy dependent $e^{+} e^{-} \rightarrow \omega \chi_{c 0}$ (left), $e^{+} e^{-} \rightarrow \eta J / \psi$ (middle) and $e^{+} e^{-} \rightarrow \eta^{\prime} J / \psi$ (right) cross sections by BESIII and Belle.

\section{The exotic $X$-states}

The unknown exotic $X$-state is always interesting and mysterious. By studying $B \rightarrow K \gamma \chi_{c 1}$ process, the Belle collaboration observed a new resonance $X(3823) \rightarrow \gamma \chi_{c 1}$ with significance $3.8 \sigma$ [प5]. The measured mass is $3823.1 \pm 1.8 \pm 0.7 \mathrm{MeV} / c^{2}$, and its width are quite narrow. Using a different approach, the BESIII collaboration studied $e^{+} e^{-} \rightarrow \pi^{+} \pi^{-} \gamma \chi_{c 1}$ process with $4.67 \mathrm{fb}^{-1}$ data above $4 \mathrm{GeV}$. By investigating the $\pi^{+} \pi^{-}$recoil system with a good resolution, the BESIII collaboration observed the $X(3823)$ resonance with $6.2 \sigma$ statistical significance [[6], and its measured mass $3821.7 \pm 1.3 \pm 0.7 \mathrm{MeV} / c^{2}$ agrees quite well with Belle. Thus, the $X(3823)$ resonance was firmly established by BESIII. Figure $⿴$ t shows the $X(3823)$ signal by Belle in $B$ meson decay and by BESIII in $e^{+} e^{-}$production. By further studying the decay ratio of $\frac{\mathscr{B}\left[X(3823) \rightarrow \gamma \chi_{c 2}\right]}{\mathscr{B}\left[X(3823) \rightarrow \gamma \chi_{c 1}\right]}$ [[15], [6]], both BESIII and Belle support it's a perfect candidate for the missing $\psi\left(1^{3} D_{2}\right)$ charmonium state. The observation of $X(3823)$ also rules out the possibility that the $X(3872)$ is the $\psi\left(1^{3} D_{2}\right)$ charmonium.
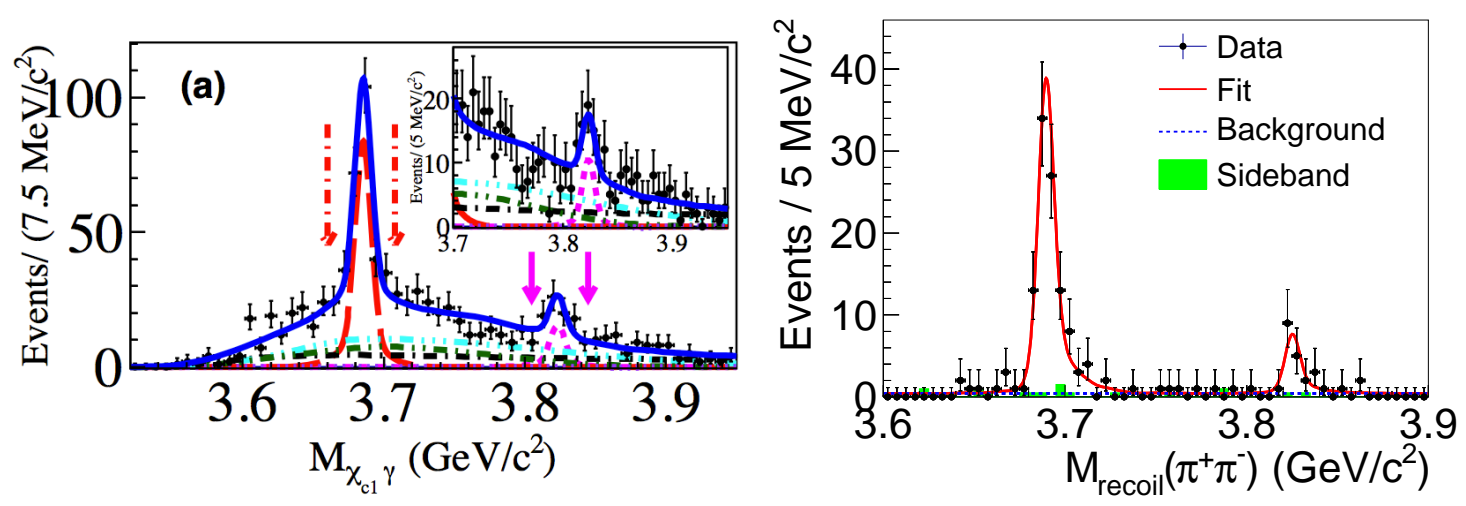

Figure 4: The measured $B^{ \pm} \rightarrow K^{ \pm} \gamma \chi_{c 1}$ mass distribution by Belle (left) and $e^{+} e^{-} \rightarrow \pi^{+} \pi^{-} \gamma \chi_{c 1}$ mass distribution by BESIII (right).

The $X(3872)$ is still the most puzzling particle more than 10 years after its discovery [D]. In order to understand its nature, various studies toward its properties are ongoing. Among these 
efforts, the BESIII collaboration's recent search for the $e^{+} e^{-} \rightarrow \gamma X(3872)$ process above $4 \mathrm{GeV}$ looks quite interesting. BESIII indeed has observed the $X(3872)$ state with $6.3 \sigma$ statistical significance [[1]], and measure its mass to be $3871.9 \pm 0.7 \pm 0.2 \mathrm{MeV} / c^{2}$, which agrees quite well with other experiments. Figure $\square$ shows the $X(3872)$ signal observed by BESIII. In addition to the $X$ (3872) observation, BESIII also measured the energy dependent production cross section of $e^{+} e^{-} \rightarrow \gamma X(3872)$, and find it strongly suggested the decay of $Y(4260) \rightarrow \gamma X(3872)$. Figure [ shows the cross section line shape of $e^{+} e^{-} \rightarrow \gamma X$ (3872) and a fit to it using $Y(4260)$ line shape. The observation of the decay $Y(4260) \rightarrow \gamma X(3872)$ for the first time brings two exotic states together, and may hint a common nature for their internal structure.
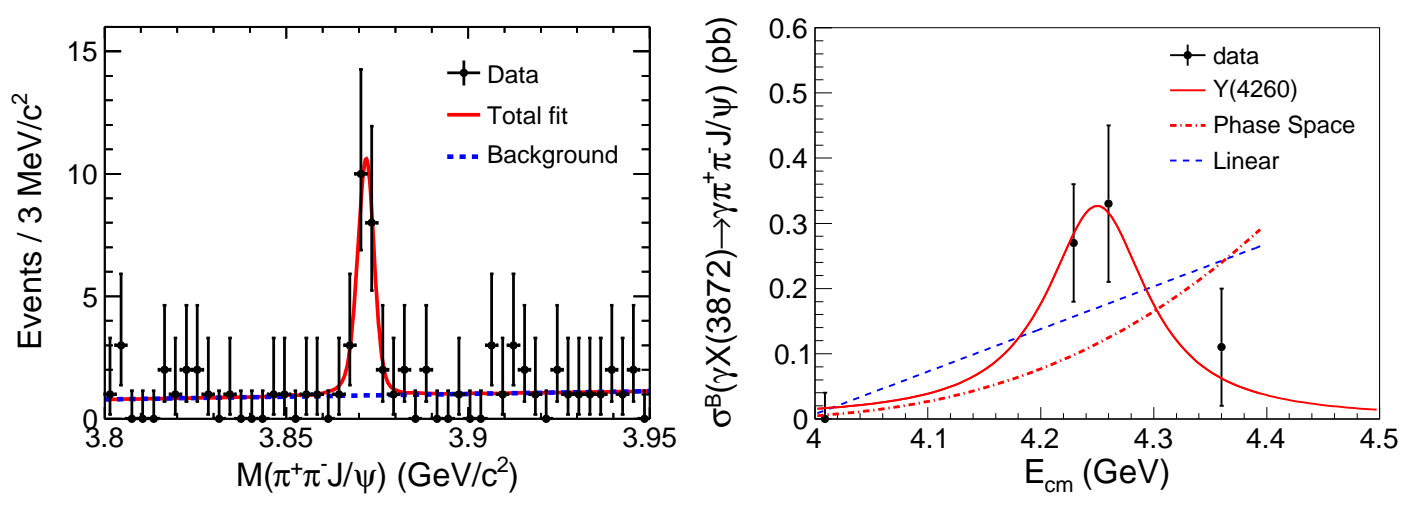

Figure 5: The $X(3872) \rightarrow \pi^{+} \pi^{-} J / \psi$ mass distribution (left) and energy dependent $e^{+} e^{-} \rightarrow \gamma X(3872)$ cross section (right) by BESIII.

\section{The charged $Z_{c}$ states}

Carrying electric charge is the obvious feature of the $Z_{c}$ states, and it definitely becomes the most important signal to distinguish $Z_{c}$ state from normal charmonium, which is neutral. In 2013, the BESIII collaboration announced the observation of a new resonance $Z_{c}(3900) \rightarrow \pi^{ \pm} J / \psi$, with mass $3899.0 \pm 3.6 \pm 4.9 \mathrm{MeV} / c^{2}$ and width $46 \pm 10 \pm 20 \mathrm{MeV}$ [5]. The Belle collaboration also report this observation at the same time, with measured mass $3894.5 \pm 6.6 \pm 4.5 \mathrm{MeV} / c^{2}$ and width $63 \pm 24 \pm 26 \mathrm{MeV}$ [四]. The $Z_{c}(3900)$ state was confirmed immediately by CELO-c data [四], with mass $3886 \pm 6 \pm 4 \mathrm{MeV} / c^{2}$ and width $33 \pm 6 \pm 7 \mathrm{MeV}$. Figure 6 shows the $Z_{c}(3900)$ signal observed by BESIII, Belle and CLEO-c's data. All the measurements from three experiments agree well with each other. Thus, the $Z_{c}(3900)$ state is the first charged charmoniumlike state to be confirmed by several experiments. $Z_{c}(3900)$ can not be normal charmonium, since it carries electric charge. The minimal quark content of $Z_{c}(3900)$ is a four quarks combination, such as $c \bar{c} u \bar{d}$.

Since $Z_{c}(3900)$ is a charged state, it's natural to think whether its neutral partner $Z_{c}(3900)^{0}$ exists. BESIII has searched for the process $e^{+} e^{-} \rightarrow \pi^{0} \pi^{0} J / \psi$, and found a neutral structure in the $\pi^{0} J / \psi$ invariant mass distribution with significance $10.4 \sigma$ [प[8]. Its mass and width are measured to be $3894.8 \pm 2.3 \pm 3.2 \mathrm{MeV} / c^{2}$ and $29.6 \pm 8.2 \pm 8.2 \mathrm{MeV}$, respectively. Figure $\square$ shows the $Z_{c}(3900)^{0}$ signal observed at three different central-of-mass energies. The observation of neutral candidate has established the iso-spin triplet of $Z_{c}(3900)$. The mass of $Z_{c}(3900)$ is quite near the 

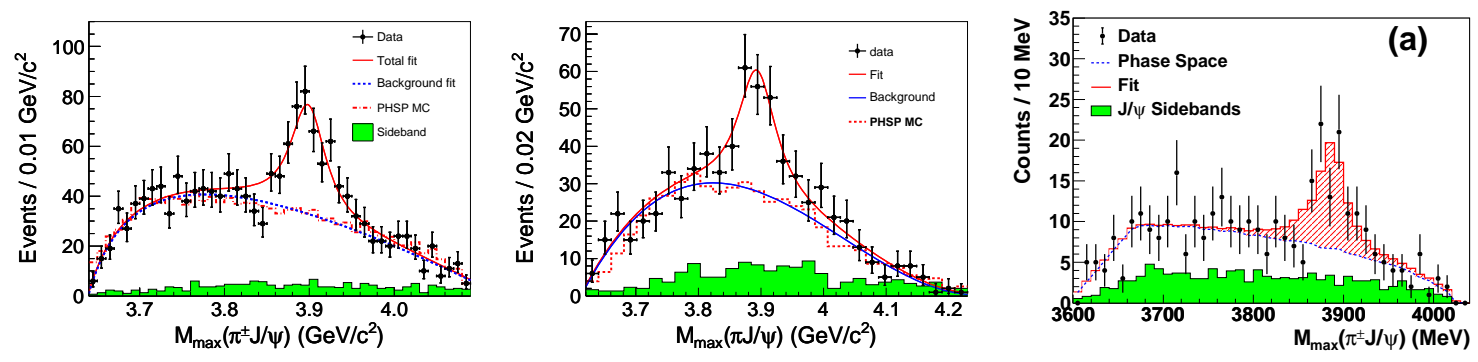

Figure 6: The $Z_{c}(3900) \rightarrow \pi^{ \pm} J / \psi$ invariant mass distribution by BESIII at $\sqrt{s}=4.26 \mathrm{GeV}$ (left), Belle using $Y(4260)$ decay (middle) and CLEO-c's data at $\sqrt{s}=4.17 \mathrm{GeV}$ (right).

threshold of $D \bar{D}^{*}$ meson pair. Thus, people are thinking whether $Z_{c}(3900)$ has a strong coupling to $D \bar{D}^{*}$ final state. Motivated by this idea, BESIII has studied the $e^{+} e^{-} \rightarrow \pi^{ \pm}\left(D \bar{D}^{*}\right)^{\mp}$ process, and an enhancement near $D \bar{D}^{*}$ threshold was observed with mass $3883.9 \pm 1.5 \pm 4.2 \mathrm{MeV} / c^{2}$ and width $24.8 \pm 3.3 \pm 1.0 \mathrm{MeV}[\mathbb{\mathbb { Q }}]$ ]. Figure $\mathrm{Z}$ shows the structure observed in $\left(D \bar{D}^{*}\right)^{ \pm}$mass distribution. It's quite possible $D \bar{D}^{*}$ is another decay channel of the $Z_{c}(3900)$ state. Taking this assumption, we also got $\Gamma\left[Z_{c}(3900) \rightarrow D \bar{D}^{*}\right] / \Gamma\left[Z_{c}(3900) \rightarrow \pi^{ \pm} J / \psi\right]=6.2 \pm 1.1 \pm 2.7$. BESIII also determined the quantum number of $Z_{c}(3900)$ to be $J^{P}=1^{+}$in $D \bar{D}^{*}$ final state by studying its scattering angular distribution.
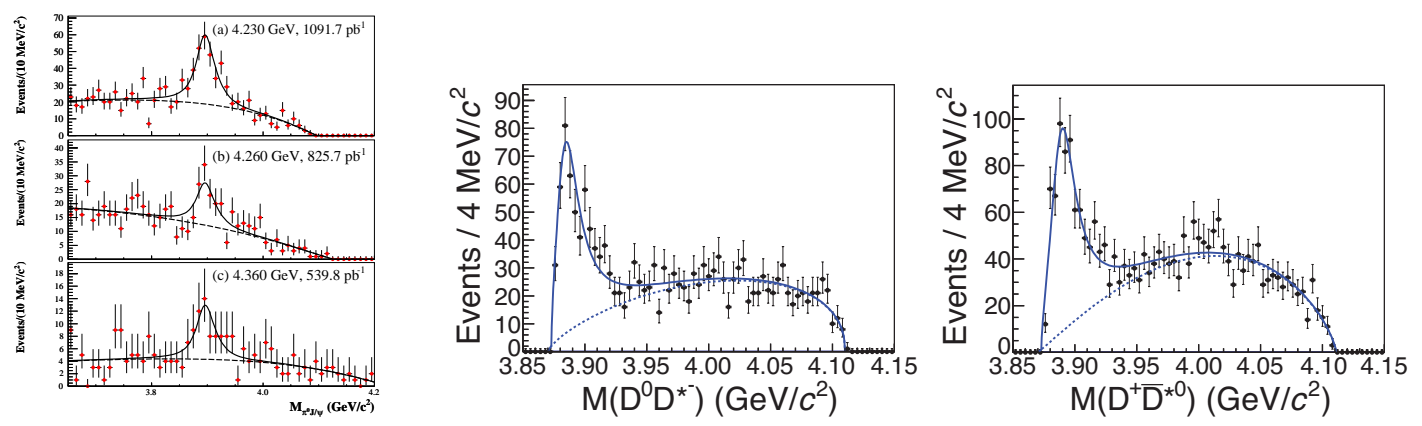

Figure 7: The $Z_{c}(3900)^{0} \rightarrow \pi^{0} J / \psi$ (left), $Z_{c}(3900) \rightarrow D^{0} D^{*-}$ (middle) and $D^{+} \bar{D}^{\bar{*} 0}$ (right) mass distribution by BESIII.

Inspired by the $Z_{c}(3900)$ discovery, BESIII also has studied the $e^{+} e^{-} \rightarrow \pi^{+} \pi^{-} h_{c}$ and $\pi^{0} \pi^{0} h_{c}$ processes. By studying the $\pi^{ \pm} h_{c}$ invariant mass distribution, a distinct structure $Z_{c}(4020)$ was observed with $>8.9 \sigma$ significance [[20]. The mass and width of this structure are measured to be $4022.9 \pm 0.8 \pm 2.7 \mathrm{MeV} / c^{2}$ and $7.9 \pm 2.7 \pm 2.6 \mathrm{MeV}$, respectively. Also by studying its isospin neutral channel, BESIII observed a neutral structure $Z_{c}(4020)^{0} \rightarrow \pi^{0} h_{c}$ with $5.0 \sigma$ significance [2]]. Its mass is $4023.9 \pm 2.2 \pm 3.8 \mathrm{MeV} / c^{2}$, which agrees with the charged partner quite well. Thus an iso-spin triplet of $Z_{c}(4020)$ was established. Figure 8 shows the charged $Z_{c}(4020)$ and neutral $Z_{c}(4020)^{0}$ signals by BESIII.

Since the mass of $Z_{c}(4020)$ was near the $D^{*} \bar{D}^{*}$ meson pair threshold, people are thinking whether it has strong coupling to $D^{*} \bar{D}^{*}$ final state. Motivated by this idea, BESIII has studied the $e^{+} e^{-} \rightarrow \pi^{ \pm}\left(D^{*} \bar{D}^{*}\right)^{\mp}$ process, and an enhancement $Z_{c}(4025)$ near $D^{*} \bar{D}^{*}$ mass threshold was observed with $>10 \sigma$ significance [[2]]. Its mass and width are measured to be $4026.3 \pm 2.6 \pm$ 

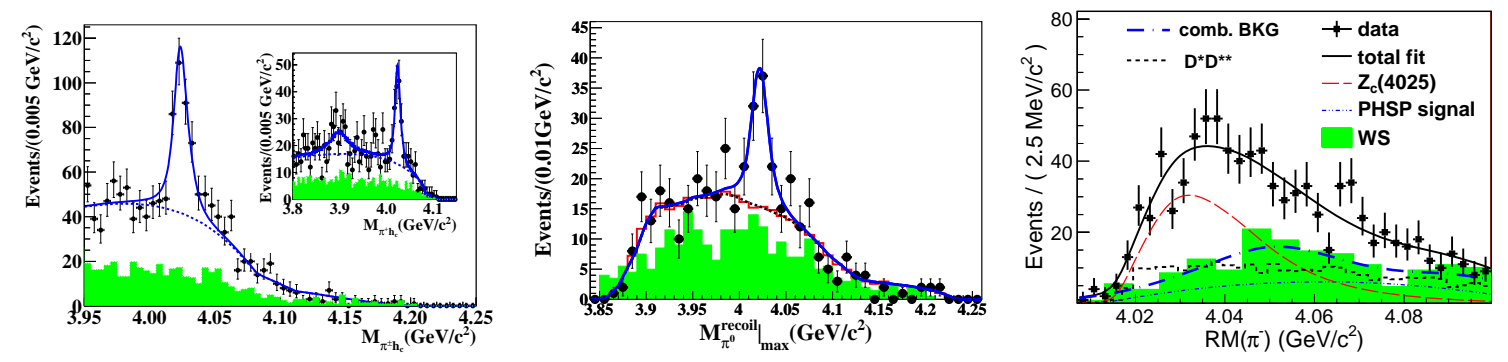

Figure 8: The $Z_{c}(4020) \rightarrow \pi^{ \pm} h_{c}$ (left), $Z_{c}(4020)^{0} \rightarrow \pi^{0} h_{c}$ (middle) and $Z_{c}(4025) \rightarrow D^{*} \bar{D}^{*}$ (right) mass distribution by BESIII.

3.7 MeV/c $c^{2}$ and $24.8 \pm 5.6 \pm 7.7 \mathrm{MeV}$, respectively. Figure $\nabla$ shows the $Z_{c}(4025)$ signal. The mass of $Z_{c}(4025)$ agrees well with $Z_{c}(4020)$, however, has a much larger width.

The Belle experiment also tries to search for charged charmoniumlike state with its huge data sets. By studying $e^{+} e^{-} \rightarrow \gamma_{I S R} \pi^{+} \pi^{-} \psi(2 S)$ process, Belle has observed resonant structures $Y(4360)$ and $Y(4660)[\mathbb{0}]$ ]. With the full data sets of $980 \mathrm{fb}^{-1}$ luminosity, Belle can probe the intermediate state in $Y(4360) \rightarrow \pi^{+} \pi^{-} \psi(2 S)$ decay. By studying the $\pi^{ \pm} \psi(2 S)$ invariant mass distribution, Belle observed a $3.5 \sigma$ evidence for the $Z_{c}(4050)$ structure, with mass $4054 \pm 3 \pm 1 \mathrm{MeV} / \mathrm{c}^{2}$ and width $45 \pm 11 \pm 6 \mathrm{MeV}$ [[23]. Figure $\mathbf{Q}$ shows the $Z_{c}(4050)$ signal, which is quite similar with the $Z_{c}(3900)$ and $Z_{c}(4020)$ observed in charged pion and charmonium final state. Belle also tries to search for charged charmoniumlike state with $B$ meson decays, such as $B^{ \pm} \rightarrow K^{ \pm} \pi^{\mp} J / \psi$ with $711 \mathrm{fb}^{-1}$ data [24]. By using amplitude analysis, Belle observed a new broad structure $Z_{c}(4200)$ with $6.2 \sigma$ significance, and also $4.0 \sigma$ evidence for the broad $Z_{c}(4430)$ structure. Figure $\mathbb{Q}$ shows the $Z_{c}(4200)$ signal, with mass $4196_{-29-13}^{+31+17} \mathrm{MeV} / c^{2}$, and width $370 \pm 70_{-132}^{+70} \mathrm{MeV}$.
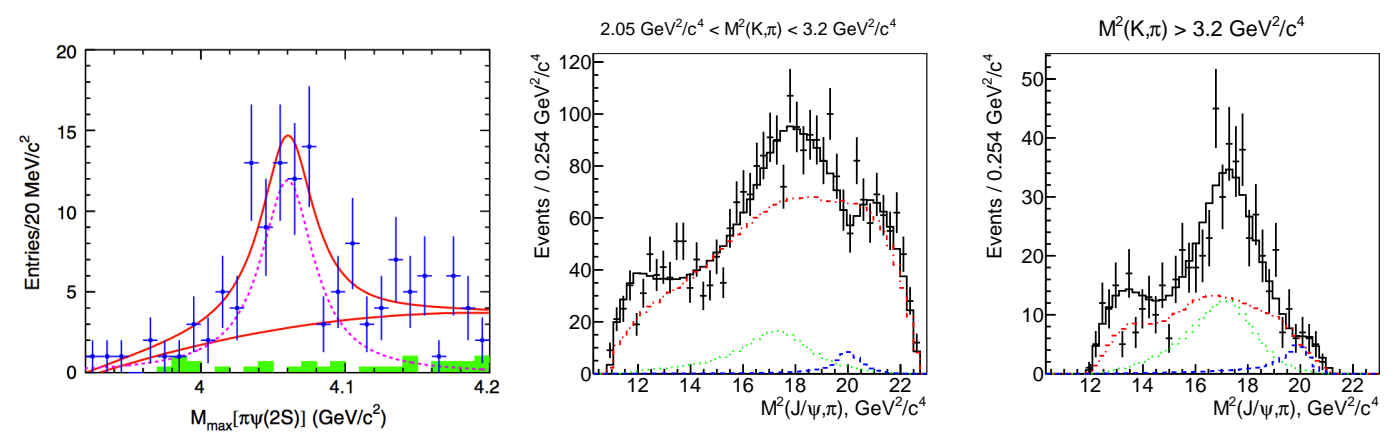

Figure 9: The $Z_{c}(4050) \rightarrow \pi^{ \pm} \psi(2 S)$ mass distribution (left) and $Z_{c}(4200) \rightarrow \pi^{ \pm} J / \psi$ mass distribution (middle and right) by Belle.

\section{Summary}

Leptonic machines always play a significant role in the studying of exotic states, with the advantage of clean background. While the $X$ and $Y$ candidates are difficult to distinguish from conventional charmonium, the charged $Z$ states are solid. However, it's still a big challenge to understand the exact quark configuration, such as tetraquark, hadron molecule and so on, inside 
these $X Y Z$ particles. The coming Belle II experiment and more data from BESIII experiment are expected to improve the current situation.

\section{References}

[1] H. Fritzsch, M. Gell-Mann and H. Leutwyler, Phys. Lett. B 47, 365 (1973).

[2] S. K. Choi et al. (Belle Collaboration), Phys. Rev. Lett. 91, 262001 (2003).

[3] B. Aubert et al. (BABAR Collaboration), Phys. Rev. Lett. 95, 142001 (2005).

[4] C. Z. Yuan et al. (Belle Collaboration) Phys. Rev. Lett. 99, 182004 (2007).

[5] M. Ablikim et al. (BESIII Collaboration), Phys. Rev. Lett. 110, 252001 (2013).

[6] Z. Q. Liu et al. (Belle Collaboration), Phys. Rev. Lett. 110, 252002 (2013).

[7] T. Xiao, S. Dobbs, A. Tomaradze, Kamal K. Seth, Phys. Lett. B 727, 366 (2013).

[8] Eric Swanson, Physics 6, 69 (2013).

[9] J. P. Lees et al. (BABAR Collaboration), Phys. Rev. D 86, 051102 (2013).

[10] X. L. Wang et al. (Belle Collaboration), Phys. Rev. Lett. 99, 142002 (2007).

[11] J. P. Lees et al. (BABAR Collaboration), Phys. Rev. D 89, 111103(R) (2014).

[12] M. Ablikim et al. (BESIII Collaboration), Phys. Rev. Lett. 114, 092003 (2015).

[13] M. Ablikim et al. (BESIII Collaboration), Phys. Rev. D 86, 071101(R) (2012); X. L. Wang et al. (Belle Collaboration), Phys. Rev. D 87, 051101 (2013); M. Ablikim et al., (BESIII Collaboration), Phys. Rev. D 91, 112005 (2015).

[14] International Workshop on Physics at Future High Intensity Collider @ 2-7GeV in China (HIEPA2014).

[15] V. Bhardwaj et al. (Belle Collaboration), Phys. Rev. Lett. 111, 032001 (2013).

[16] M. Ablikim et al. (BESIII Collaboration), Phys. Rev. Lett. 115, 011803 (2015).

[17] M. Ablikim et al. (BESIII Collaboration), Phys. Rev. Lett. 112, 092001 (2014).

[18] M. Ablikim et al. (BESIII Collaboration), arXiv:1506.06018.

[19] M. Ablikim et al. (BESIII Collaboration), Phys. Rev. Lett. 112, 022001 (2014).

[20] M. Ablikim et al. (BESIII Collaboration), Phys. Rev. Lett. 111, 242001 (2013).

[21] M. Ablikim et al. (BESIII Collaboration), Phys. Rev. Lett. 113, 212002 (2014).

[22] M. Ablikim et al. (BESIII Collaboration), Phys. Rev. Lett. 112, 132001 (2014).

[23] X. L. Wang et al. (Belle Collaboration), Phys. Rev. D 91, 112007 (2015).

[24] K. Chilikin et al. (Belle Collaboration), Phys. Rev. D 90, 112009 (2014). 